Rabaska

Revue d'ethnologie de l'Amérique française

\title{
Chaire de recherche du Canada sur les migrations, les transferts et les communautés francophones (Université de Saint-Boniface)
}

\section{Yves Frenette et Yves Labrèche}

Volume 12, 2014

URI : https://id.erudit.org/iderudit/1026836ar

DOI : https://doi.org/10.7202/1026836ar

Aller au sommaire du numéro

Éditeur(s)

Société québécoise d'ethnologie

ISSN

1703-7433 (imprimé)

1916-7350 (numérique)

Découvrir la revue

Citer ce document

Frenette, Y. \& Labrèche, Y. (2014). Chaire de recherche du Canada sur les migrations, les transferts et les communautés francophones (Université de Saint-Boniface). Rabaska, 12, 354-356. https://doi.org/10.7202/1026836ar d'utilisation que vous pouvez consulter en ligne. 


\section{OuesT}

\section{Chaire de recherche du Canada sur les migrations, les transferts et les communautés francophones}

Université de Saint-Boniface

Téléphone : (204) 237-1818, poste 237

200, avenue de la Cathédrale

Courriel :yfrenette@ustboniface.ca

Saint-Boniface (Manitoba)

Toile : http://ustboniface.ca/crc-mtcf

$\mathrm{R} 2 \mathrm{H} 0 \mathrm{H} 7$

\section{Présentation}

Créée à l'automne 2013, grâce à un octroi du programme des Chaires de recherche du Canada, la Chaire de recherche du Canada de niveau 1 sur les migrations, les transferts et les communautés francophones ( $\mathrm{CRC}-\mathrm{MTCF}$ ) vise à promouvoir la recherche sur les francophonies des Amériques en mettant l'accent sur les phénomènes migratoires depuis l'établissement des premiers colons d'origine française au Nouveau Monde. Le programme insiste également sur les transferts au sens le plus large : éducation, transmission du patrimoine culturel et linguistique, acculturation et interculturalité. Dans un contexte où les migrations transnationales contribuent de façon marquée à la recomposition des communautés francophones contemporaines, l'existence d'une chaire universitaire dont l'objet est la compréhension des phénomènes et des processus migratoires s'avère essentielle dans le paysage scientifique nord-américain.

\section{Ancrage de la chaire}

La Crc-MTcF est logée à l'Université de Saint-Boniface (Winnipeg, Manitoba), au cœur de l'Amérique francophone pendant toute la durée du programme (2013-2020). Les travaux de la CRC-MTCF viennent renforcer la zone de concentration en recherche de cet établissement qui touche les enjeux culturels et identitaires des francophones et des Métis. Les perspectives diachroniques et ethnohistoriques de cette nouvelle chaire permettent d'embrasser le passé lointain des francophones des Amériques autant que l'histoire contemporaine qui se construit sous nos yeux au Manitoba ainsi que dans d'autres régions du Canada, des États-Unis et de l'Amérique latine. La CRCMTCF est à développer un réseau de partenaires à partir de l'Université qui l'abrite.

\section{Chantiers}

Pour réaliser les deux volets de recherche de la CRC-MTCF, soit les migrations francophones historiques et les migrations francophones contemporaines, le titulaire Yves Frenette, le coordonnateur Yves Labrèche (ylabreche@ustboniface.ca) et leurs associés développent une méthodologie appropriée à chacun d'entre eux.

A - Perspective diachronique, 1680-1970 - Mise sur pied ou continuation de huit chantiers spatiotemporels qui, chacun, permettent d'analyser un mouvement ou un contexte migratoire spécifique dans le temps et l'espace. Les huit chantiers sont : Cap-Santé, 1680-1970, vieille localité sur les rives du Saint-Laurent ; Saint-Boniface, 
1750-1970, porte d'entrée de l'Ouest francophone ; Dakota du Nord, 1750-1940, région commerciale puis agricole du Midwest des États-Unis ; Bas-Caraquet et Haut-Caraquet, 1755-1880, deux localités voisines peuplées par des groupes de francophones distincts ; l'est de l'Ontario, 1820-1960, région agro-forestière voisine du Québec et où est située la capitale du Canada ; Lewiston, 1860-1900, centre textile de la Nouvelle-Angleterre ; immigration française au Canada entre 1870 et 1914, chantier qui permet de comparer les migrants dans différentes destinations ; Montréal-Sao Paulo, 1895-1897, épisode migratoire hémisphérique. Pour chaque chantier, le titulaire, le coordonnateur et les associés de recherche dépouillent les sources manuscrites et imprimées disponibles ; ils recueillent aussi des données quantitatives sur les migrants.

Les recherches effectuées dans le cadre des huit chantiers alimentent des bases de données nominatives constituées à partir d'informations puisées dans les recensements, registres d'état civil, outils généalogiques, bottins et autres sources quantitatives. À terme, cela permettra de dresser le profil différencié des migrants acadiens, canadiens-français, métis et franco-européens entre le milieu du XVII ${ }^{\mathrm{e}}$ siècle et le troisième quart du $\mathrm{xx}^{\mathrm{e}}$ siècle. Pour la première fois, l'établissement d'un tel profil permettra de faire des généralisations, explicitement, sur les migrations francophones et de les comparer, implicitement, aux mouvements migratoires d'autres groupes. Pour comprendre les transferts culturels qui sont inhérents aux migrations et qui ont un impact sur la genèse et l'évolution des communautés francophones, le titulaire et plusieurs cochercheurs continuent à recueillir et à analyser des correspondances dans les diasporas francophones des Amériques. Parallèlement, la CRC-MTCF est à mettre sur pied un projet de compilation et d'analyse des manuels scolaires en Amérique française du XVII ${ }^{\mathrm{e}}$ siècle à nos jours.

B - Perspective contemporaine, depuis 1970 - Pour documenter et analyser les migrations francophones contemporaines, le titulaire, le coordonnateur et leurs associés de recherche ont recours à la méthodologie de l'histoire orale. Dans le but d'appréhender ces mouvements migratoires dans leur entièreté et dans leur diversité, ils réalisent des entrevues en tenant compte des lieux d'origine des migrants, de leur appartenance culturelle, des types de migration, du genre, de la classe sociale et des lieux d'implantation.

\section{Activités de diffusion}

La CRC-MTCF a instauré dès sa première année un programme de conférences annuelles pour honorer la mémoire de Robert Painchaud, un historien, décédé prématurément en 1977, qui a contribué de manière significative à la connaissance de l'histoire de Saint-Boniface et du Manitoba, notamment en ce qui a trait à l'étude du peuplement francophone. La première de ces conférences annuelles a eu lieu en mars 2014, lors de la soirée d'inauguration de la CRC-MTсF. Le professeur Luca Codignola-Bo de l'Université de Genova (Italie) a présenté alors une conférence intitulée « Rome et les débuts de Saint-Boniface (1818-1836) ». La deuxième conférence annuelle Robert-Painchaud aura lieu au début d'octobre 2014 et sera prononcée par le professeur Hans-Jürgen Lüsebrink, de l’Université de Saarbrücken (Allemagne); 
elle portera sur les almanachs canadiens-français et insistera sur l'histoire ainsi que l'impact socioculturel de ce média populaire.

D'autres conférences se sont tenues régulièrement dans le cadre des activités de la CRC-MTCF : ainsi, le professeur Gérard Fabre (CNRS et ÉHess) a prononcé le 15 avril 2014 une conférence sur le roman Un homme se penche sur son passé de Maurice Constantin-Weyer, Prix Goncourt 1928. Deux jours plus tard, le professeur Annick Foucrier (Université Paris 1, Panthéon-Sorbonne) présenta ses recherches sur les Français et Canadiens de Californie pendant la période de la Ruée vers l'or.

Par ailleurs, depuis son entrée en fonction le $1^{\mathrm{er}}$ août 2013, Yves Frenette, titulaire de la CRC-MTCF, a poursuivi ses recherches, tout en participant à plusieurs colloques au Canada et à l'étranger. En octobre-novembre, il a occupé la Chaire d'étude sur le Canada Pres Limousin-Poitou-Charentes et, en novembre-décembre, il a été professeur étranger invité à l'École nationale des chartes.

En outre, grâce à l'appui du coordonnateur de la CRC-MTCF et d'un soutien technique de l'Université de Saint-Boniface, la chaire a mis sur pied un site au cours de sa première année d'existence. Enfin, elle organise les 28, 29 et 30 août 2015 à l'Université de Saint-Boniface un colloque international et interdisciplinaire sur les écrits personnels et les sources orales en relation avec la mobilité, du $\mathrm{XIV}^{\mathrm{E}}$ au XXI siècle.

Yves FrenetTe

YVEs LABRÈCHE 